TRANSACTIONS OF THE

AMERICAN MATHEMATICAL SOCIETY

Volume 349, Number 9, September 1997, Pages 3803-3821

S 0002-9947(97)01978-8

\title{
A CONSTRUCTION OF CODIMENSION THREE ARITHMETICALLY GORENSTEIN SUBSCHEMES OF PROJECTIVE SPACE
}

\author{
JUAN C. MIGLIORE AND CHRIS PETERSON
}

\begin{abstract}
This paper presents a construction method for a class of codimension three arithmetically Gorenstein subschemes of projective space. These schemes are obtained from degeneracy loci of sections of certain specially constructed rank three reflexive sheaves. In contrast to the structure theorem of Buchsbaum and Eisenbud, we cannot obtain every arithmetically Gorenstein codimension three subscheme by our method. However, certain geometric applications are facilitated by the geometric aspect of this construction, and we discuss several examples of this in the final section.
\end{abstract}

\section{INTRODUCTION}

A projective scheme $V \subset \mathbb{P}^{n}$ is said to be arithmetically Gorenstein (or projectively Gorenstein) if its homogeneous coordinate ring is a Gorenstein ring. Arithmetically Gorenstein schemes provide a generalization of complete intersection schemes, and in fact the two classes of schemes coincide in codimension two. They have appeared as an important component in a number of problems and have proven useful in a wide variety of applications. We will illustrate one such application in the final section of this paper by constructing several low degree, complex threefolds in $\mathbb{P}^{6}$.

We are going to approach arithmetically Gorenstein schemes from a geometric viewpoint by producing them as sections of certain specially constructed sheaves. Let $\mathcal{F}$ be a coherent sheaf on $\mathbb{P}^{n}$. The dual, $\mathcal{F}^{\vee}$, of $\mathcal{F}$ is defined by $\mathcal{F}^{\vee}=$ $\mathcal{H o m}\left(\mathcal{F}, \mathcal{O}_{\mathbb{P}^{n}}\right)$. The sheaf $\mathcal{F}$ is said to be reflexive if $\mathcal{F} \simeq \mathcal{F}^{\vee}$. Reflexive sheaves provide a natural generalization of holomorphic vector bundles, and it is from this viewpoint that we proceed. Complete intersection schemes arise as the vanishing locus of sections of a special class of vector bundles, namely those bundles which split as the direct sum of line bundles. Thus, the special properties of the sections of these vector bundles reflect the special properties of the vector bundles themselves. Following this philosophy, we present a method for constructing codimension three arithmetically Gorenstein subschemes of $\mathbb{P}^{n}$. We restrict our attention to a special class of rank three reflexive sheaves, namely those with vanishing in certain "higher cohomology modules". The arithmetically Gorenstein schemes are then obtained as

Received by the editors April 30, 1996.

1991 Mathematics Subject Classification. Primary 14F05, 14M05; Secondary 14M06, 14M07, $13 \mathrm{D} 02$.

Key words and phrases. Rank three reflexive sheaves, codimension three schemes, arithmetically Gorenstein, linkage, liaison, Buchsbaum-Rim complex.

(C)1997 American Mathematical Society 
the top dimensional component of the vanishing locus of sections of these sheaves. The main theorem that we prove is the following.

Theorem 1.1. Let $R=k\left[x_{0}, \ldots, x_{n}\right]$ be a polynomial ring over the algebraically closed field $k$. Let $A$ be a $t \times(t+3)$ homogeneous matrix defined over $R$ such that the ideal of $t \times t$ minors of $A$ defines a scheme of codimension four. View $A$ as defining a map between two free $R$-modules $F$ and $G$. Let $Q$ denote the kernel of this map. Sheafify $Q$ to obtain a rank three reflexive sheaf $\mathcal{Q}$. Let $s$ be a section of $\mathcal{Q}$ which has a codimension three degeneracy locus, $S$. Let $I_{X}$ denote the saturated homogeneous ideal of the pure codimension three component of $S$. Let $c_{1}$ denote the first Chern class of $\mathcal{Q}$. Then $I_{X}$ is arithmetically Gorenstein with free resolution

$$
0 \rightarrow R\left(-c_{1}\right) \rightarrow \underset{\substack{F\left(-c_{1}\right) \\ G^{\vee}}}{\rightarrow} \underset{\substack{G\left(-c_{1}\right) \\ F^{\vee}}}{\rightarrow} I_{X} \rightarrow 0 .
$$

With a small amount of work, the theorem has a particularly nice form in $\mathbb{P}^{3}$.

Corollary 1.2. Let $\mathcal{E}$ be a rank three vector bundle in $\mathbb{P}^{3}$ such that $H^{2}\left(\mathbb{P}^{3}, \mathcal{E}(n)\right)=$ 0 for all $n$. Let $s$ be a section of $\mathcal{E}$ vanishing on a codimension three scheme $X$. Then $X$ is arithmetically Gorenstein.

In section 2 of the paper, we describe the setting and prove some initial results. Section 3 considers the special case of arithmetically Gorenstein sets of points in $\mathbb{P}^{3}$, while section 4 considers the general case of codimension three arithmetically Gorenstein schemes in $\mathbb{P}^{n}$.

In the final section we first present the main application that motivated our investigations: Gorenstein linkage theory. Gorenstein linkage theory is the study of a certain equivalence relation on the class of equidimensional subschemes of $\mathbb{P}^{n}$. To be effective in a wide variety of applications, Gorenstein linkage requires the construction of arithmetically Gorenstein schemes with certain prescribed properties (e.g. low degree and containment of a given scheme). Buchsbaum and Eisenbud proved a beautiful structure theorem for codimension three arithmetically Gorenstein schemes. They were able to show that the homogeneous ideal of every such scheme arises as the Pfaffian of a skew symmetric matrix. This has been an extremely helpful viewpoint, but its applications to linkage theory have been somewhat limited due to the difficulty in arranging the properties mentioned above (see for instance Examples 5.3, 5.4). One advantage of the geometric aspect of the construction presented in this paper is that codimension three Gorenstein linkage theory becomes much more practical.

A natural question is whether all codimension three arithmetically Gorenstein schemes can be obtained by this construction. We answer this question in the negative by producing an example of a non-reduced scheme supported on a point, which cannot be so obtained. We close the paper with an application to threefolds in $\mathbb{P}^{6}$, and a discussion of open problems related to constructions in higher codimension.

We would like to thank the referee for making several helpful remarks.

\section{General Set-up}

Let $R=k\left[x_{0}, x_{1}, \ldots, x_{n}\right]$ be a graded ring, where $k$ is an algebraically closed field with characteristic zero and $n \geq 3$. Let $F$ be a graded free $R$-module of rank $t+3$ and let $G$ be a graded free $R$-module of rank $t$. A homogeneous matrix is 
a matrix in which every minor is a homogeneous polynomial. Maps from $F$ to $G$ are given by $t \times(t+3)$ homogeneous matrices. The degrees of the entries of the matrix are such that the map is given as a graded degree zero homomorphism. For example, if $F=\bigoplus_{i=1}^{t+3} R\left(a_{i}\right)$ and $G=\bigoplus_{j=1}^{t} R\left(b_{j}\right)$, then any map from $F$ to $G$ will be given by a $t \times(t+3)$ matrix $A$. The $c_{i j}$ entry of $A$ will be either zero or a homogeneous polynomial of degree $b_{i}-a_{j}$. Now let's view things in the other direction. Let $A$ be a general $t \times(t+3)$ homogeneous matrix. Such a matrix defines a map between two graded free $R$-modules. Let $I_{j}(A)$ denote the ideal consisting of all $j \times j$ minors of $A$ (also called the $(t-j)^{t h}$ Fitting ideal of $A$ ). One expects $I_{t}(A)$ to define a codimension four subscheme of $\mathbb{P}^{n}$. These are precisely the conditions we need for our construction. Henceforth, we assume that $A$ is an arbitrary $t \times(t+3)$ homogeneous matrix defined over $R=k\left[x_{0}, x_{1}, \ldots, x_{n}\right]$ such that $I_{t}(A)$ defines a scheme, $P$, of codimension 4 in $\mathbb{P}^{n}$. Let $Q$ denote the kernel of the map defined by $A$ and let $\operatorname{coker}(A)$ denote the cokernel of the map. We thus have the following exact sequence:

$$
0 \rightarrow Q \rightarrow F \stackrel{A}{\rightarrow} G \rightarrow \operatorname{coker}(A) \rightarrow 0 .
$$

Note that if $n=3$ then $\operatorname{coker}(A)$ is a graded module of finite length.

Let $K$ denote the cokernel of the map from $Q$ to $F$ (or equivalently the kernel of the map from $G$ to $\operatorname{coker}(A))$. We can break up (2.1) into two short exact sequences:

$$
\begin{gathered}
0 \rightarrow Q \rightarrow F \rightarrow K \rightarrow 0, \\
0 \rightarrow K \rightarrow G \rightarrow \operatorname{coker}(A) \rightarrow 0 .
\end{gathered}
$$

Sheafify (2.2) to yield an exact sequence of sheaves.

$$
0 \rightarrow \mathcal{Q} \rightarrow \mathcal{F} \rightarrow \mathcal{K} \rightarrow 0 .
$$

If $n=3$, then $\mathcal{Q}$ is locally free and $\mathcal{K}$ is free (in fact $\mathcal{K}=\mathcal{G}$, where $\mathcal{G}$ is the sheafification of $G$ ). In general, $\mathcal{Q}$ has rank three and is reflexive since it is locally a second syzygy sheaf. Define $H_{*}^{i}\left(\mathbb{P}^{n}, \mathcal{E}\right)=\bigoplus_{t \in \mathbb{Z}} H^{i}\left(\mathbb{P}^{n}, \mathcal{E}(t)\right)$. By the BuchsbaumRim complex [18], $H_{*}^{i}\left(\mathbb{P}^{n}, \mathcal{Q}\right)=0$ for $1 \leq i \leq n-3$ and $H_{*}^{0}\left(\mathbb{P}^{n}, \mathcal{Q}\right)=Q$ if $n \geq 3$. Without loss of generality, we can assume that $\mathcal{Q}$ is twisted sufficiently so as to have sections. Let $s$ be a section of $\mathcal{Q}$ which has a codimension three degeneracy locus. $s$ determines the sequence

$$
0 \rightarrow \mathcal{O}_{\mathbb{P}^{n}} \stackrel{s}{\rightarrow} \mathcal{Q} \rightarrow \mathcal{C} \rightarrow 0
$$

where $\mathcal{C}$ denotes the quotient of $\mathcal{Q}$ by the section defined by $s$. Taking global sections yields the exact sequence of graded $R$-modules

$$
0 \rightarrow R \stackrel{s}{\rightarrow} Q \rightarrow C \rightarrow 0
$$

Proposition 2.1. The sheaf $\mathcal{C}$ is reflexive.

Proof. Let $s^{t}$ denote the dual of the map defined by $s$. Let $S$ denote the degeneracy locus of $s$. By assumption, $S$ is a scheme of codimension three. Let $\mathcal{O}_{S}$ denote the structure sheaf of $S$ and let $\mathcal{I}_{S}$ denote the ideal sheaf of $S$. By [33], p. 152, we have the useful fact that $\mathcal{E} x t_{\mathcal{O}_{\mathbb{P} n}}^{1}\left(\mathcal{C}, \mathcal{O}_{\mathbb{P}^{n}}\right)=\mathcal{O}_{S}$. Using this identification, we dualize (2.5) to obtain

$$
0 \rightarrow \mathcal{C}^{\vee} \rightarrow \mathcal{Q}^{\vee} \stackrel{s^{t}}{\rightarrow} \mathcal{O}_{\mathbb{P}^{n}} \rightarrow \mathcal{O}_{S} \rightarrow 0
$$


Hence we have the exact sequence

$$
0 \rightarrow \mathcal{C}^{\vee} \rightarrow \mathcal{Q}^{\vee} \rightarrow \mathcal{I}_{S} \rightarrow 0 .
$$

Since $\mathcal{I}_{S}$ is the ideal sheaf of a codimension three scheme, $\mathcal{E} x t_{\mathcal{O}_{\mathbb{P}} n}^{1}\left(\mathcal{I}_{S}, \mathcal{O}_{\mathbb{P}^{n}}\right)=0$. Dualizing (2.8) and using the fact that $\mathcal{H}_{o m_{\mathcal{O}^{n}}}\left(\mathcal{I}_{S}, \mathcal{O}_{\mathbb{P}^{n}}\right)=\mathcal{O}_{\mathbb{P}^{n}}$, we obtain the sequence

$$
0 \rightarrow \mathcal{O}_{\mathbb{P}^{n}} \rightarrow \mathcal{Q}^{\vee \vee} \rightarrow \mathcal{C}^{\vee \vee} \rightarrow 0
$$

Since $\mathcal{Q}$ is reflexive, $\mathcal{Q}^{\vee \vee}=\mathcal{Q}$. This forces $\mathcal{C}^{\vee \vee}=\mathcal{C}$.

Note that the scheme $S$ defined above is not equidimensional (except in $\mathbb{P}^{3}$ ). The scheme $S$ consists of an equidimensional codimension three component as well as a codimension four component arising from the support of $\operatorname{coker}(A)$. Let $X$ denote the pure codimension three component of $S$. In $\mathbb{P}^{3}, X$ and $S$ are equal. In $\mathbb{P}^{4}, X$ is a scheme of pure dimension one and hence is locally Cohen-Macaulay. From the exact sequence $(2.5)$ we see that $\mathcal{C}$ has rank two. We will have use of the following

Lemma 2.2. Let $\mathcal{D}$ be a rank two reflexive sheaf. Let $c_{1}$ be the first Chern class of $\mathcal{D}$. Then there is an isomorphism $\mathcal{D}^{\vee} \simeq \mathcal{D}\left(-c_{1}\right)$.

Proof. See [20].

Since $\mathcal{C}$ is a rank two reflexive sheaf, we utilize Lemma 2.2 to rewrite sequence (2.8) as

$$
0 \rightarrow \mathcal{C}\left(-c_{1}\right) \rightarrow \mathcal{Q}^{\vee} \rightarrow \mathcal{I}_{S} \rightarrow 0 .
$$

We are now in a position to prove the main proposition of this section.

Proposition 2.3. If $n \geq 4$ then $H_{*}^{1}\left(\mathbb{P}^{n}, \mathcal{I}_{S}\right) \simeq H_{*}^{1}\left(\mathbb{P}^{n}, \mathcal{K}\left(-c_{1}\right)\right)$.

Proof. To begin with, we sheafify exact sequence (2.3). This leads to the exact sequence of sheaves

$$
0 \rightarrow \mathcal{K} \rightarrow \mathcal{G} \rightarrow \mathcal{W} \rightarrow 0
$$

where $\mathcal{W}$ denotes the sheafification of $\operatorname{coker}(A)$. Since $\mathcal{W}$ is supported on a codimension four scheme, we have

$$
\mathcal{H o m}_{\mathcal{O}_{\mathbb{p}} n}\left(\mathcal{W}, \mathcal{O}_{\mathbb{P}^{n}}\right)=\mathcal{E} x t_{\mathcal{O}_{\mathbb{p}} n}^{1}\left(\mathcal{W}, \mathcal{O}_{\mathbb{P}^{n}}\right)=\mathcal{E} x t_{\mathcal{O}_{\mathbb{p}} n}^{2}\left(\mathcal{W}, \mathcal{O}_{\mathbb{P}^{n}}\right)=0
$$

[19]. Dualizing exact sequence (2.11) and utilizing the vanishing of the aforementioned $\mathcal{E} x$ groups, we obtain that $\mathcal{K}^{\vee} \simeq \mathcal{G}^{\vee}$ and that $\mathcal{E} x t_{\mathcal{O}_{\mathbb{P}}}^{1}\left(\mathcal{K}, \mathcal{O}_{\mathbb{P}^{n}}\right)=0$. We now dualize (2.4) and utilize these facts to obtain

$$
0 \rightarrow \mathcal{G}^{\vee} \rightarrow \mathcal{F}^{\vee} \rightarrow \mathcal{Q}^{\vee} \rightarrow 0 .
$$

Since $\mathcal{F}$ and $\mathcal{G}$ are free, so are $\mathcal{F}^{\vee}$ and $\mathcal{G}^{\vee}$; hence we can use (2.12) to show that $H_{*}^{1}\left(\mathbb{P}^{n}, \mathcal{Q}^{\vee}\right)=H_{*}^{2}\left(\mathbb{P}^{n}, \mathcal{Q}^{\vee}\right)=0$ (since $\left.n \geq 4\right)$. From $(2.10)$, we now obtain that $H_{*}^{1}\left(\mathbb{P}^{n}, \mathcal{I}_{S}\right) \simeq H_{*}^{2}\left(\mathbb{P}^{n}, \mathcal{C}\left(-c_{1}\right)\right)$. Finally, from $(2.5)$ we obtain that $H_{*}^{2}\left(\mathbb{P}^{n}, \mathcal{C}\left(-c_{1}\right)\right) \simeq$ $H_{*}^{2}\left(\mathbb{P}^{n}, \mathcal{Q}\left(-c_{1}\right)\right)$ and from $(2.4)$ we obtain that $H_{*}^{2}\left(\mathbb{P}^{n}, \mathcal{Q}\left(-c_{1}\right)\right) \simeq H_{*}^{1}\left(\mathbb{P}^{n}, \mathcal{K}\left(-c_{1}\right)\right)$. Hence $H_{*}^{1}\left(\mathbb{P}^{n}, \mathcal{I}_{S}\right) \simeq H_{*}^{1}\left(\mathbb{P}^{n}, \mathcal{K}\left(-c_{1}\right)\right)$.

Remark 2.4. The sheaf $\mathcal{W}$ is supported on a codimension four scheme. Hence for $n \geq 4$ we have $H_{*}^{n-3}\left(\mathbb{P}^{n}, \mathcal{W}\right)=H_{*}^{n-2}\left(\mathbb{P}^{n}, \mathcal{W}\right)=H_{*}^{n-1}\left(\mathbb{P}^{n}, \mathcal{W}\right)=H_{*}^{n}\left(\mathbb{P}^{n}, \mathcal{W}\right)=$ 0 . From $(2.11)$ we obtain that $H_{*}^{n-2}\left(\mathbb{P}^{n}, \mathcal{K}\right)=H_{*}^{n-1}\left(\mathbb{P}^{n}, \mathcal{K}\right)=0$. Finally, from (2.4) we obtain that $H_{*}^{n-1}\left(\mathbb{P}^{n}, \mathcal{Q}\right)=0$. In $\mathbb{P}^{3}, \mathcal{K}$ is free; hence we easily obtain $H_{*}^{2}\left(\mathbb{P}^{n}, \mathcal{Q}\right)=0$. Altogether, for $n \geq 3$ we have $H_{*}^{n-1}\left(\mathbb{P}^{n}, \mathcal{Q}\right)=0$. 


\section{Gorenstein Points in $\mathbb{P}^{3}$}

In this section we prove that if $n=3$ then the codimension three scheme, $X$, that we obtained in the previous section is arithmetically Gorenstein. (Notice that $X=S$ in this case; see section 2 for notation.) In some sense this is the heart of the paper, since the higher dimensional cases are proved by reducing to this case. More precisely, we prove:

Theorem 3.1. Let $A$ be a $t \times(t+3)$ homogeneous matrix over $R=k\left[x_{0}, x_{1}, x_{2}, x_{3}\right]$ such that the ideal of $t \times t$ minors of $A$ is $\left(x_{0}, x_{1}, x_{2}, x_{3}\right)$-primary. View $A$ as defining a map between two graded free R-modules $F$ and $G$. Let $Q$ denote the kernel of this map. Sheafify $Q$ to obtain a rank three locally free sheaf $\mathcal{Q}$. Let $s$ be a section of $\mathcal{Q}$ which has a codimension three degeneracy locus, $S$. Let $I_{S}$ denote the saturated homogeneous ideal of $S$. Let $c_{1}$ denote the first Chern class of $\mathcal{Q}$. Then $I_{S}$ is arithmetically Gorenstein with free resolution

$$
0 \rightarrow R\left(-c_{1}\right) \rightarrow \begin{gathered}
F\left(-c_{1}\right) \\
\underset{G^{\vee}}{\oplus}
\end{gathered} \rightarrow \underset{\substack{G\left(-c_{1}\right) \\
F^{\vee}}}{\rightarrow} \rightarrow I_{S} \rightarrow 0 .
$$

Proof. We will construct a free resolution for the saturated ideal $I_{X}$. This resolution will be "almost minimal," and we then apply a result of Kreuzer to deduce the Gorenstein property. This allows us to split off one more term, resulting in the resolution given above.

Consider a minimal free resolution of $H_{*}^{1}\left(\mathbb{P}^{3}, \mathcal{Q}\right)$,

$$
0 \rightarrow F_{4} \rightarrow F_{3} \rightarrow F_{2} \rightarrow F \rightarrow G \rightarrow H_{*}^{1}\left(\mathbb{P}^{3}, \mathcal{Q}\right) \rightarrow 0 .
$$

(The entire resolution can be obtained by the Buchsbaum-Rim complex.) We then have a commutative diagram of sheaves

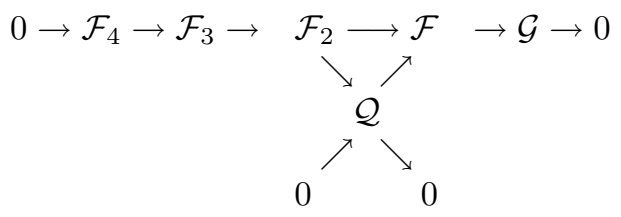

From sequences (2.6) and (3.2) we get a diagram

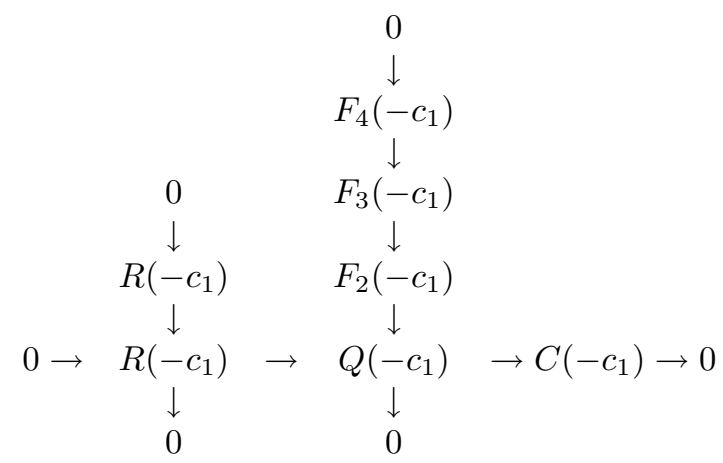

from which a mapping cone gives

$$
0 \rightarrow F_{4}\left(-c_{1}\right) \rightarrow R\left(-c_{1}\right) \oplus F_{3}\left(-c_{1}\right) \rightarrow F_{2}\left(-c_{1}\right) \rightarrow C\left(-c_{1}\right) \rightarrow 0 .
$$


Notice also that (3.2) gives

$$
0 \rightarrow G^{\vee} \rightarrow F^{\vee} \rightarrow H_{*}^{0}\left(\mathbb{P}^{3}, \mathcal{Q}^{\vee}\right) \rightarrow 0 .
$$

Now, from the long exact sequence in cohomology associated to (2.10) and using the facts that $H_{*}^{1}\left(\mathbb{P}^{3}, \mathcal{Q}^{\vee}\right)=0$ and $X=S$, we get

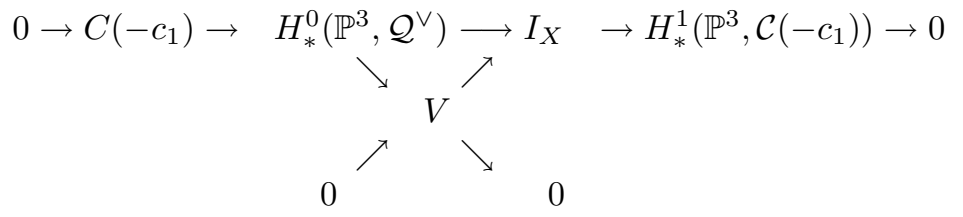

where $V$ is simply the cokernel of the first map. Notice, however, that the saturation of $V$ as an $R$-module is $I_{X}$, since $H_{*}^{1}\left(\mathbb{P}^{3}, \mathcal{C}\left(-c_{1}\right)\right)$ has finite length. The mapping cone applied to the leftmost short exact sequence in (3.5), and using the resolutions (3.3) and (3.4), gives the exact sequence

$$
0 \rightarrow F_{4}\left(-c_{1}\right) \rightarrow R\left(-c_{1}\right) \oplus F_{3}\left(-c_{1}\right) \rightarrow F_{2}\left(-c_{1}\right) \oplus G^{\vee} \rightarrow F^{\vee} \rightarrow V \rightarrow 0 .
$$

Now consider the free resolution

$0 \rightarrow F_{4}\left(-c_{1}\right) \rightarrow F_{3}\left(-c_{1}\right) \rightarrow F_{2}\left(-c_{1}\right) \rightarrow F\left(-c_{1}\right) \rightarrow G\left(-c_{1}\right) \rightarrow H_{*}^{1}\left(\mathbb{P}^{3}, \mathcal{Q}\right)\left(-c_{1}\right) \rightarrow 0$.

Since $H_{*}^{1}\left(\mathbb{P}^{3}, \mathcal{Q}\right)=H_{*}^{1}\left(\mathbb{P}^{3}, \mathcal{C}\right)$, we get from (3.5), (3.6) and (3.7) the following diagram:

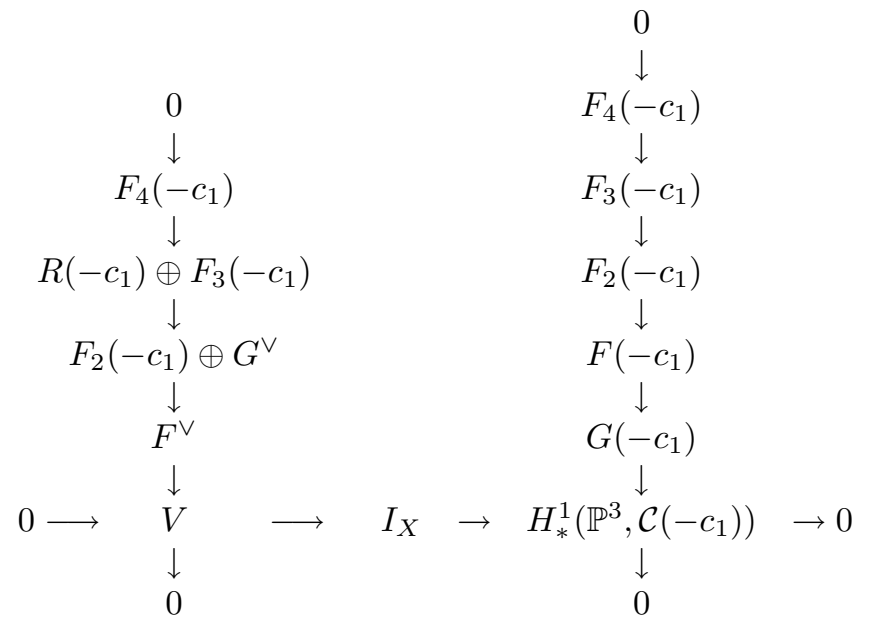

The Horseshoe Lemma ([44], 2.2.8, p. 37) then gives the following free resolution for the saturated ideal $I_{X}$ :

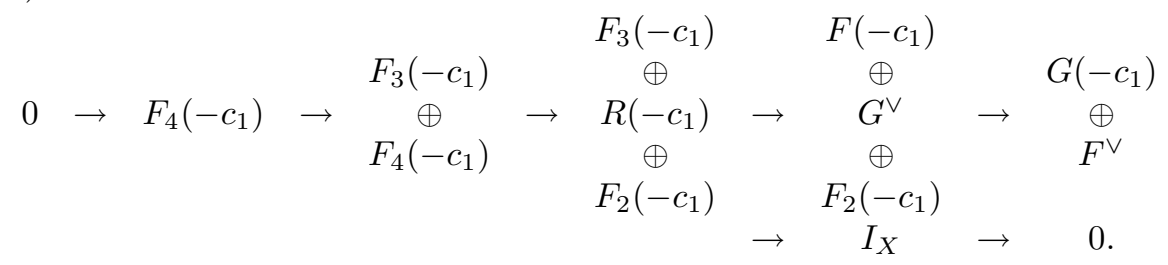


Since $I_{X}$ has projective dimension 2, the maps $F_{4}\left(-c_{1}\right) \rightarrow F_{4}\left(-c_{1}\right)$ and $F_{3}\left(-c_{1}\right) \rightarrow$ $F_{3}\left(-c_{1}\right)$ are isomorphisms, so we can split off these terms to get

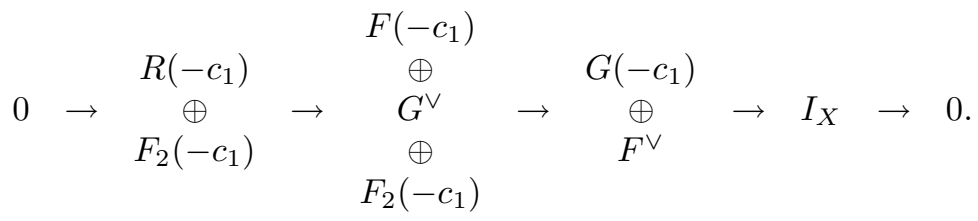

It is clear that the two copies of $F_{2}\left(-c_{1}\right)$ in (3.10) do not contribute to a calculation of the Hilbert function of $R / I_{X}$, nor to the Hilbert function of the canonical module, $\omega_{X}$, of $X$ (since (3.10) is a resolution of the saturated homogeneous ideal of $X$, so we can dualize and shift to obtain a free resolution of $\omega_{X}$ ). It then follows from the form of (3.10) that the first difference of the Hilbert function of $R / I_{X}$ is symmetric. On the other hand, Kreuzer [25] has generalized the result of Griffiths and Harris ([17], Proposition 1.22), and has shown that $X$ (reduced or not) has the Cayley-Bacharach property with respect to the linear system $\left|K_{\mathbb{P}^{3}} \otimes \operatorname{deg} Q\right|$. (We would like to thank Mark De Cataldo for showing us the reference [17], Proposition 1.22.) Finally, by [24], Theorem 1.1 (which in turn is a generalization to the non-reduced case of [10], Theorem 5), these two properties imply that $X$ is arithmetically Gorenstein. This means that the Cohen-Macaulay type of $I_{X}$ is one, and so by the symmetry of the resolution we can split off the $F_{2}\left(-c_{1}\right)$, obtaining the claimed resolution for $I_{X}$.

Corollary 3.2. Let $s$ be a section of a rank three vector bundle $\mathcal{Q}$ on $\mathbb{P}^{3}$ with vanishing second cohomology, $H_{*}^{2}\left(\mathbb{P}^{3}, \mathcal{Q}\right)=0$, but not vanishing first cohomology, and assume that $s$ vanishes on a zero-scheme $X$. Then $X$ is arithmetically Gorenstein.

Proof. Consider a minimal free resolution of $H_{*}^{1}\left(\mathbb{P}^{3}, \mathcal{Q}\right)$ :

$$
0 \rightarrow F_{4} \rightarrow F_{3} \rightarrow F_{2} \rightarrow F \rightarrow G \rightarrow H_{*}^{1}\left(\mathbb{P}^{3}, \mathcal{Q}\right) \rightarrow 0 .
$$

Sheafifying, we get

$$
0 \rightarrow \mathcal{F}_{4} \rightarrow \mathcal{F}_{3} \rightarrow \mathcal{F}_{2} \rightarrow \mathcal{F} \stackrel{\alpha}{\rightarrow} \mathcal{G} \rightarrow 0
$$

We claim that the kernel of $\alpha$ is $\mathcal{Q}$. Indeed, by [11], 1.5, since $\mathcal{Q}$ has the same first and second cohomology as $\operatorname{ker} \alpha$ we get that $\mathcal{Q}=\operatorname{ker} \alpha \oplus \mathcal{L}$ for some free bundle $\mathcal{L}$. But $\mathcal{Q}$ is indecomposable, by an easy application of the Evans-Griffith syzygy theorem [14], and so we are done. Hence $\mathcal{Q}$ is the kernel of a surjection $\mathcal{F} \rightarrow \mathcal{G}$ of free sheaves, and since rank $\mathcal{Q}=3$, we have the conditions needed for Theorem 3.1.

From Corollary 3.2 one is led to the natural question of whether similar results might hold for a vector bundle $\mathcal{Q}$ with vanishing first cohomology. It turns out that we do not, in general, get an arithmetically Gorenstein zero-scheme, but we can nevertheless find a free resolution for the saturated ideal of the resulting zeroscheme. Note that sequences (2.5), (2.6), and (2.10) still apply.

Theorem 3.3. Let $\mathcal{Q}$ be a rank three vector bundle on $\mathbb{P}^{3}$ with vanishing first cohomology, $H_{*}^{1}\left(\mathbb{P}^{3}, \mathcal{Q}\right)=0$, but not vanishing second cohomology. Consider the minimal free resolution of $H_{*}^{2}\left(\mathbb{P}^{3}, \mathcal{Q}\right)$,

$$
0 \rightarrow F_{4} \rightarrow F_{3} \rightarrow F_{2} \rightarrow F_{1} \rightarrow F_{0} \rightarrow H_{*}^{2}\left(\mathbb{P}^{3}, \mathcal{Q}\right) \rightarrow 0 .
$$


The sheaf $\mathcal{Q}$ arises as the sheafification of the cokernel of the map from $F_{4}$ to $F_{3}$. Let $s$ be a section of $\mathcal{Q}$ and assume that $s$ vanishes on a zero-scheme $S$. Then $I_{S}$ has a free resolution

$$
0 \rightarrow R\left(-c_{1}\right) \oplus F_{4}\left(-c_{1}\right) \oplus F_{0}^{\vee} \rightarrow F_{3}\left(-c_{1}\right) \oplus F_{1}^{\vee} \rightarrow F_{2}^{\vee} \rightarrow I_{S} \rightarrow 0 .
$$

Proof. It is easy to show that $\mathcal{Q}$ is indecomposable. We start with the minimal free resolution of $H_{*}^{2}\left(\mathbb{P}^{3}, \mathcal{Q}\right)$,

$$
0 \rightarrow F_{4} \rightarrow F_{3} \rightarrow F_{2} \rightarrow F_{1} \rightarrow F_{0} \rightarrow H_{*}^{2}\left(\mathbb{P}^{3}, \mathcal{Q}\right) \rightarrow 0 .
$$

Sheafifying, we get

$$
0 \rightarrow \mathcal{F}_{4} \stackrel{\alpha}{\rightarrow} \mathcal{F}_{3} \rightarrow \mathcal{F}_{2} \rightarrow \mathcal{F}_{1} \rightarrow \mathcal{F}_{0} \rightarrow 0
$$

As above, one can verify that the cokernel of $\alpha$ is $\mathcal{Q}$. Hence we have an exact diagram

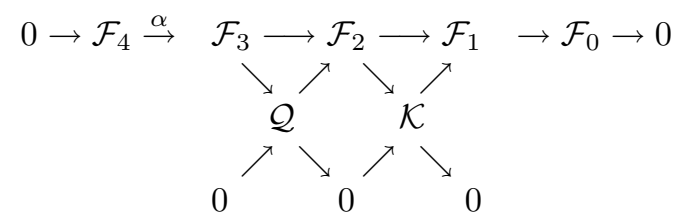

where $\mathcal{K}$ is locally free.

Notice that we also get a minimal free resolution

$$
0 \rightarrow F_{4} \rightarrow F_{3} \rightarrow H_{*}^{0}\left(\mathbb{P}^{3}, \mathcal{Q}\right) \rightarrow 0 .
$$

Hence from the sequence (2.6) and using a mapping cone argument, we get a resolution

$$
0 \rightarrow R\left(-c_{1}\right) \oplus F_{4}\left(-c_{1}\right) \rightarrow F_{3}\left(-c_{1}\right) \rightarrow C\left(-c_{1}\right) \rightarrow 0 .
$$

Claim. We have a minimal free resolution

$$
0 \rightarrow F_{0}^{\vee} \rightarrow F_{1}^{\vee} \rightarrow F_{2}^{\vee} \rightarrow H_{*}^{0}\left(\mathbb{P}^{3}, \mathcal{Q}^{\vee}\right) \rightarrow 0 .
$$

From the diagram (3.11) we get

$$
0 \rightarrow \mathcal{F}_{0}^{\vee} \rightarrow \mathcal{F}_{1}^{\vee} \rightarrow \mathcal{K}^{\vee} \rightarrow 0
$$

and hence $H_{*}^{1}\left(\mathbb{P}^{3}, \mathcal{K}^{\vee}\right)=0$. It also follows from this sequence that at the module level we have the resolution

$$
0 \rightarrow F_{0}^{\vee} \rightarrow F_{1}^{\vee} \rightarrow H_{*}^{0}\left(\mathbb{P}^{3}, \mathcal{K}^{\vee}\right) \rightarrow 0 .
$$

Hence we get the diagram

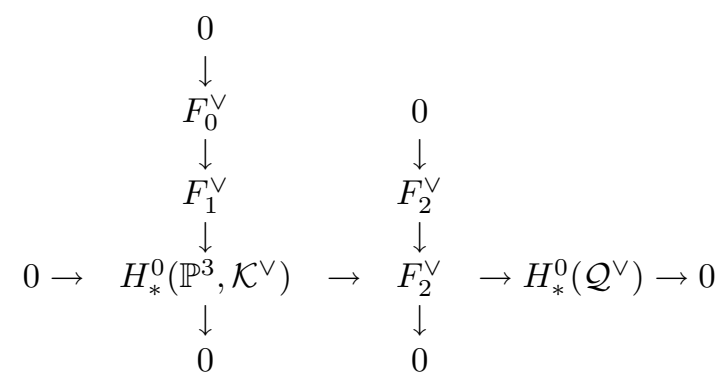

Taking a mapping cone of this diagram and noticing that the matrices obtained are the transposes of the original matrices in the minimal free resolution of $H_{*}^{2}\left(\mathbb{P}^{3}, \mathcal{Q}\right)$ proves the claim. 
Now, from $(2.10)$ and using the fact that $H_{*}^{1}\left(\mathbb{P}^{3}, \mathcal{C}\right)=0,(3.12)$ and $(3.13)$ lead to the diagram

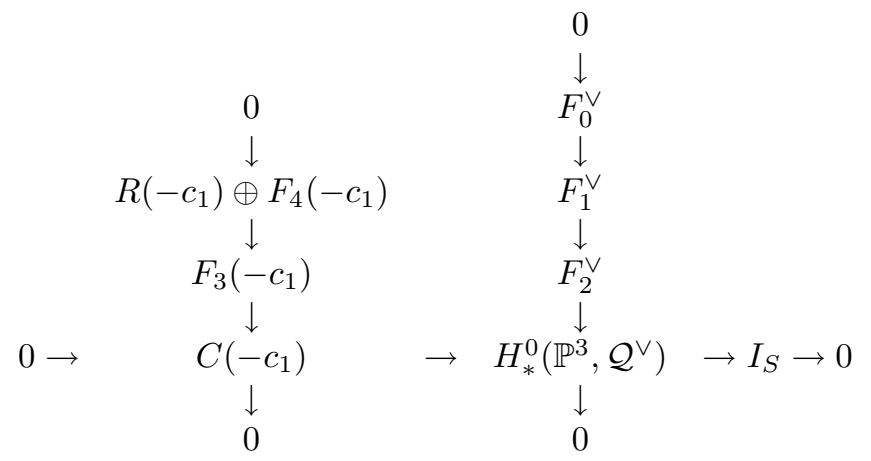

and the mapping cone gives a free resolution

$$
0 \rightarrow R\left(-c_{1}\right) \oplus F_{4}\left(-c_{1}\right) \oplus F_{0}^{\vee} \rightarrow F_{3}\left(-c_{1}\right) \oplus F_{1}^{\vee} \rightarrow F_{2}^{\vee} \rightarrow I_{S} \rightarrow 0
$$

Notice that in this proof it was not necessary to saturate the ideal defined by the section: the fact that $H_{*}^{1}\left(\mathbb{P}^{3}, \mathcal{C}\right)=0$ guarantees that this ideal is already saturated.

\section{Constructing Codimension Three Arithmetically Gorenstein Subschemes of $\mathbb{P}^{n}$}

In this section we prove the main result of this paper, namely the following theorem:

Theorem 4.1. Let $A$ be a $t \times(t+3)$ homogeneous matrix over $R$ such that the ideal of $t \times t$ minors of $A$ defines a scheme of codimension four. View $A$ as defining a degree zero map between two graded free $R$-modules $F$ and $G$. Let $Q$ denote the kernel of this map. Sheafify $Q$ to obtain a rank three reflexive sheaf $\mathcal{Q}$. Let $s$ be a section of $\mathcal{Q}$ which has a codimension three degeneracy locus, $S$. Let $I_{X}$ denote the homogeneous ideal of the pure codimension three component of $S$. Let $c_{1}$ denote the first Chern class of $\mathcal{Q}$. Then $I_{X}$ is arithmetically Gorenstein with free resolution

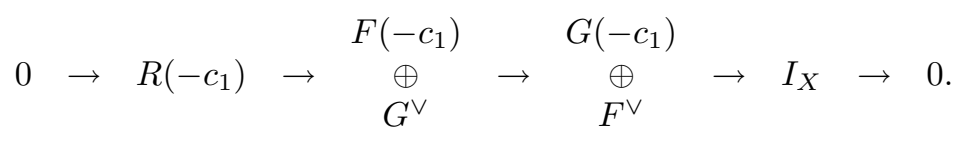

Proof. Let $\mathcal{K}$ denote the image of $A$. Note that $\mathcal{K}$ is a subsheaf of $\mathcal{G}$. We have seen that as long as $n \geq 4$, we have $H_{*}^{1}\left(\mathbb{P}^{n}, \mathcal{Q}\right)=0$ (this follows from the Buchsbaum-Rim complex $)$ and $H_{*}^{2}\left(\mathbb{P}^{n}, \mathcal{Q}\right) \cong H_{*}^{1}\left(\mathbb{P}^{n}, \mathcal{K}\right)$. Also, for any $n \geq 3$, we have $H_{*}^{n-1}\left(\mathbb{P}^{n}, \mathcal{Q}\right)=$ 0. 
Let $H$ be a general hyperplane. From the commutative diagram

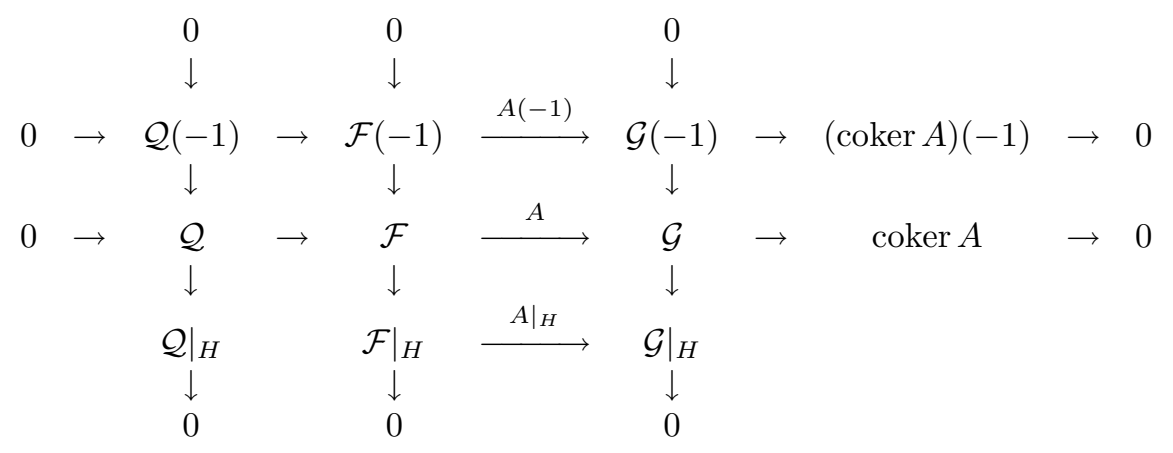

we see that $\left.\mathcal{Q}\right|_{H}$ is the kernel of $\left.A\right|_{H}$. Since $H$ is general, $\left.A\right|_{H}$ drops rank on a scheme of (the expected) codimension four. Hence we can use an inductive argument.

We begin by assuming that $n \geq 5$. A section $s$ of $\mathcal{Q}$ defines a scheme, $S$, of codimension three. Let $X$ be the top dimensional component of $S$. We claim that $X$ is arithmetically Cohen-Macaulay. This follows from the fact that the restriction of $s$ to $H$ is a section of $\left.\mathcal{Q}\right|_{H}$ which drops rank on the scheme $S \cap H$, and the top dimensional component of $S \cap H$ is $X \cap H$. By induction we may assume that $X \cap H$ is arithmetically Gorenstein, hence in particular arithmetically Cohen-Macaulay. Since $X \cap H$ has dimension $\geq 1$, it follows that $X$ itself is arithmetically CohenMacaulay ([30], Remark 1.10, [41], Teorema 2, or [23], Proposition 2.1, the latter being somewhat more general than we need here). Since the Cohen-Macaulay type is invariant under general hyperplane section, $X$ is arithmetically Gorenstein.

To complete the proof we have to begin the induction. The case $n=3$ was done in the last section. We have only to prove the case $n=4$. From now on we assume this, so $P$ is a zero-scheme in $\mathbb{P}^{4}$, and both $S$ and $X$ are curves in $\mathbb{P}^{4}$.

Claim 1. Let $L$ be a general linear form. Then the map induced by $L$ on first cohomology,

$$
\times L: H^{1}\left(\mathbb{P}^{4}, \mathcal{K}(t-1)\right) \rightarrow H^{1}\left(\mathbb{P}^{4}, \mathcal{K}(t)\right),
$$

is surjective for any $t$. Hence the same is true for

$$
\times L: H^{2}\left(\mathbb{P}^{4}, \mathcal{Q}(t-1)\right) \rightarrow H^{2}\left(\mathbb{P}^{4}, \mathcal{Q}(t)\right) .
$$

To see this, let $H$ be the hyperplane defined by $L$. Consider the exact sequence

$$
0 \rightarrow \mathcal{K} \rightarrow \mathcal{G} \rightarrow \mathcal{W} \rightarrow 0 .
$$

Since $\mathcal{W}$ (i.e. the sheafification of $\operatorname{coker}(A))$ is supported on the zero-scheme $P$, we see that as long as $H$ avoids $P$ we get $\left.\left.\mathcal{K}\right|_{H} \cong \mathcal{G}\right|_{H}$. (In particular, $\left.A\right|_{H}$ is surjective.) On the other hand, since $L$ is general we have a short exact sequence

$$
\left.0 \rightarrow \mathcal{K}(-1) \rightarrow \mathcal{K} \rightarrow \mathcal{K}\right|_{H} \rightarrow 0 .
$$

Taking cohomology and using the fact that $\left.\left.\mathcal{K}\right|_{H} \cong \mathcal{G}\right|_{H}$ completes the proof of Claim 1.

Note that in any case $X$ is locally Cohen-Macaulay and equidimensional, and $S$ is supported on the union of $X$ and $P$. Also, we have

$$
H_{*}^{1}\left(\mathbb{P}^{4}, \mathcal{I}_{S}\right)=H_{*}^{1}\left(\mathbb{P}^{4}, \mathcal{K}\right)\left(-c_{1}\right) .
$$


Observe that the restriction $\left.\mathcal{Q}\right|_{H}$ of $\mathcal{Q}$ to a general hyperplane $H=\mathbb{P}^{3}$ is locally free, since the singular locus of $Q$ is zero-dimensional. The restriction of $s$ to $H$ is a section of this locally free sheaf on $H$, and the zero-scheme it defines is the hyperplane section $Z$ of $S$. Since $H$ avoids $P, Z$ is also the hyperplane section of $X$. From the exact sequence

$$
\left.0 \rightarrow \mathcal{Q}(-1) \rightarrow \mathcal{Q} \rightarrow \mathcal{Q}\right|_{H} \rightarrow 0
$$

together with the discussion above of the cohomology of $\mathcal{Q}$ (including Claim 1), we get that $H_{*}^{2}\left(\mathbb{P}^{4},\left.\mathcal{Q}\right|_{H}\right)=0$. From Corollary 3.2 we then see that $Z$ is arithmetically Gorenstein.

Our task here is to show that $X$ is arithmetically Gorenstein. As before, this will follow once we show that $X$ is arithmetically Cohen-Macaulay, since the CohenMacaulay type is preserved taking hyperplane sections of arithmetically CohenMacaulay subschemes of projective space.

We can write the homogeneous ideal of $S$ as $I_{S}=I_{X} \cap \mathfrak{q}$, where $\mathfrak{q}$ is the saturated ideal of some zero-scheme $P_{1}$ supported on the support of $P$. We thus have an exact sequence

$$
0 \rightarrow I_{S} \rightarrow I_{X} \oplus \mathfrak{q} \rightarrow I_{X}+\mathfrak{q} \rightarrow 0
$$

It is clear that $I_{X}+\mathfrak{q}$ is the (not necessarily saturated) ideal of a zero-scheme $P_{2}$, which is a subscheme of $P_{1}$.

Note that $H$ avoids $P_{1}$. Sheafifying, we get the commutative diagram

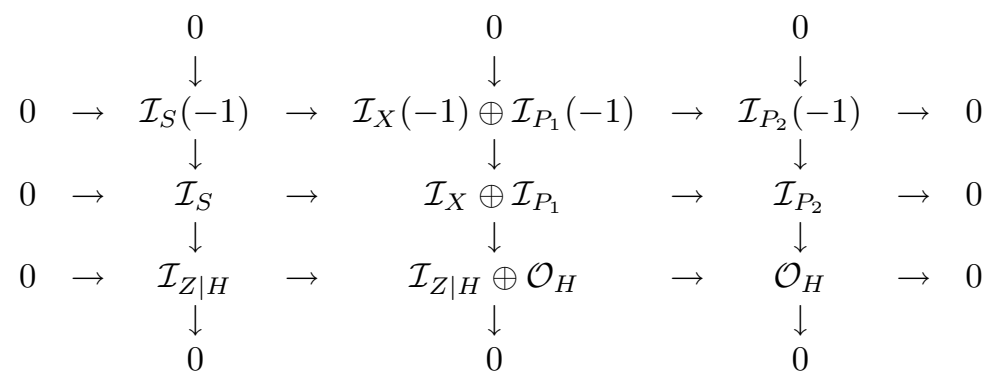

Note that if $P_{2}$ is empty, then $I_{P_{2}}=R$.

Claim 2. We have an isomorphism of $R$-modules, $H_{*}^{2}\left(\mathbb{P}^{4}, \mathcal{I}_{S}\right) \cong H_{*}^{2}\left(\mathbb{P}^{4}, \mathcal{I}_{X}\right)$.

To see this, consider the exact sequence

$$
0 \rightarrow \mathcal{I}_{S} \rightarrow \mathcal{I}_{X} \rightarrow \mathcal{W} \rightarrow 0
$$

where $\mathcal{W}$ is some sheaf supported on a zero-scheme. Hence

$$
H_{*}^{1}\left(\mathbb{P}^{4}, \mathcal{W}\right)=H_{*}^{2}\left(\mathbb{P}^{4}, \mathcal{W}\right)=0
$$

so taking cohomology on the above sequence gives the desired result and proves Claim 2. 
Using Claim 2, we get the following commutative diagram in cohomology from the diagram (4.3):

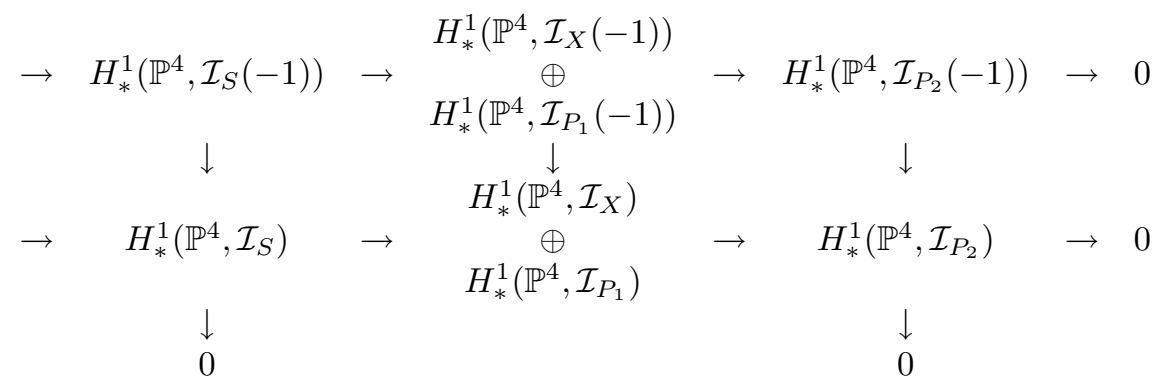

(The first vertical surjection comes from (4.2) and Claim 1.) It then follows that

$$
H_{*}^{1}\left(\mathbb{P}^{4}, \mathcal{I}_{X}(-1)\right) \rightarrow H_{*}^{1}\left(\mathbb{P}^{4}, \mathcal{I}_{X}\right)
$$

is a surjection. But $X$ is a locally Cohen-Macaulay, equidimensional curve, so this module has finite length. Hence this map cannot be surjective unless the module is zero. Therefore $X$ is arithmetically Cohen-Macaulay, and hence arithmetically Gorenstein, as desired.

Corollary 4.2. Let $\mathcal{Q}$ be a rank three reflexive sheaf on $\mathbb{P}^{n}$ with vanishing higher cohomology modules, $H_{*}^{r}\left(\mathbb{P}^{n}, \mathcal{Q}\right)=0$ for $2 \leq r \leq n-1$, but not vanishing first cohomology. Let $s$ be a section of $\mathcal{Q}$ which vanishes on a scheme, $S$, of codimension three. Then the top dimensional component of $S$ is arithmetically Gorenstein.

Remark 4.3. It is an interesting application of the paper [23] to observe that "most of the time" the curve $X$ discussed in the proof of Theorem 4.1 is automatically arithmetically Gorenstein just from the fact that its hyperplane section, $Z$, is. Indeed, if $X$ is reduced and connected and lies on no quadric hypersurface, then we are done. However, the proof given requires no such assumptions on $X$.

\section{Closing Remarks and Examples}

5.1. Linkage. One of the most promising applications of our construction is in the area of linkage, or liaison, theory. Schenzel has shown [42] that if we link using arithmetically Gorenstein schemes rather than complete intersection schemes, the same invariants arise (see also [7]). So, using this fact, we make the "naive" definition that two schemes are (directly) Gorenstein linked if their union is an arithmetically Gorenstein scheme. (The naivity of this definition arises when the schemes have a common component, and in this case there is an algebraic version which eliminates the problem.)

In codimension two, the properties of being arithmetically Gorenstein and being a complete intersection are equivalent. In this case, liaison theory is extremely rich in terms of classification theorems and structure theorems (e.g. [1], [7], [28], [34], [39], [40]). This has resulted in a wealth of applications. It has been important, for instance, in the classification and construction of smooth curves in $\mathbb{P}^{3}$ (e.g. [6], [15], [21], [27]), smooth surfaces in $\mathbb{P}^{4}$ (e.g. [5], [12], [36], [38]) and smooth threefolds in $\mathbb{P}^{5}$ (e.g. [3], [4], [12], [29]). Combined with results in intersection theory, it has also facilitated the construction of low-rank vector bundles (e.g. [35]). (These lists are very far from complete. See [31] for an in-depth discussion of liaison theory in codimension two, including applications.) 
In higher codimension the set of complete intersection schemes and the set of arithmetically Gorenstein schemes do not coincide. The set of complete intersection schemes forms a proper subset of the set of arithmetically Gorenstein schemes. To date, liaison theory has not proved as useful in constructing examples as it has in codimension two. Perhaps the most interesting application of liaison to codimension three is the paper [32]. We believe that the principal reason that liaison has not proved useful in codimension three (apart from [32]) is that it is too restrictive to consider only complete intersections. The main obstacle to extending this is that with present tools, it is hard to find an arithmetically Gorenstein ideal contained in a given ideal (of the same codimension), apart from the complete intersections (where one simply has to look for a regular sequence of the right length).

The authors plan to develop, in a forthcoming paper, the machinery to apply the construction of this paper to liaison theory so that Gorenstein linkage will begin to have results (at least in codimension three) analogous to those in codimension two, and so that applications to the classification of codimension three varieties can be begun in a way analogous to that which has proved so fruitful in codimension two. But we would like to show, in this subsection, how to use our construction to perform Gorenstein linkage. Even if the method we describe now is far from being the best possible for finding Gorenstein links, in this section we show that it already gives a different equivalence relation than is obtained using only complete intersections. In the next subsection we use this naive idea to construct some new smooth threefolds in $\mathbb{P}^{6}$.

The idea is simple, and we illustrate it for the case of zero-schemes in $\mathbb{P}^{3}$. It is well known that if one restricts to complete intersection linkage, then there is not a unique linkage class. Indeed, it was pointed out to the first author by B. Ulrich that a set $Z$ of four points in linear general position is not in the linkage class of a complete intersection - this follows from [22], Proposition 4.12. On the other hand, one can quickly see that $Z$ is in the same linkage class as a complete intersection if we instead use Gorenstein linkage. Indeed, it follows from Theorem 5 of [10] that five points in linear general position are arithmetically Gorenstein, so $Z$ is Gorenstein linked to one point.

We now show how to produce this link using the construction of this paper. Begin with the cotangent bundle $\Omega_{\mathbb{P}^{3}}$. Note that $\Omega_{\mathbb{P}^{3}}$ satisfies the hypothesis of Corollary 3.2 on cohomology. From the exact sequence

$$
0 \rightarrow \Omega_{\mathbb{P}^{3}}(3) \rightarrow \mathcal{O}_{\mathbb{P}^{3}}(2)^{4} \rightarrow \mathcal{O}_{\mathbb{P}^{3}}(3) \rightarrow 0
$$

we get $h^{0}\left(\mathbb{P}^{3}, \Omega_{\mathbb{P}^{3}}(3)\right)=20$. A Chern class computation shows that $c_{3}\left(\Omega_{\mathbb{P}^{3}}(3)\right)=5$. Since $\Omega_{\mathbb{P}^{3}}(2)$ is globally generated, a general section of $\Omega_{\mathbb{P}^{3}}(3)$ vanishes on five points. The sequence above comes from the sheafification of the minimal free resolution of the one-dimensional vector space $k$, so a section of $H^{0}\left(\mathbb{P}^{3}, \Omega_{\mathbb{P}^{3}}(3)\right)$ can be viewed as a linear combination (using linear forms as coefficients) of the columns of the matrix of Koszul relations on $(w, x, y, z)$

$$
\left[\begin{array}{rrrrrr}
-x & -y & -z & 0 & 0 & 0 \\
w & 0 & 0 & -y & -z & 0 \\
0 & w & 0 & x & 0 & -z \\
0 & 0 & w & 0 & x & y
\end{array}\right]
$$

Hence such a section may be viewed as a four-tuple of quadrics. Vanishing at a general point of $\mathbb{P}^{3}$ imposes at most three conditions on sections of $H^{0}\left(\mathbb{P}^{3}, \Omega_{\mathbb{P}^{3}}(3)\right)$, 
so there are sections which vanish at the four points of $Z$. In fact, it is easy to show that if $\mathcal{I}_{P}$ represents the ideal sheaf of four general points in $\mathbb{P}^{3}$, then

$$
h^{0}\left(\mathbb{P}^{3}, \Omega_{\mathbb{P}^{3}}(3) \otimes \mathcal{I}_{P}\right)=8 .
$$

To show that the general such section vanishes in codimension three, one can either make a direct argument (for instance begin by assuming that $Z$ consists of the four coordinate points), or more simply, rely on the computer to produce an example. Either way, one can then use Corollary 3.2 and Theorem 4.1 to compute that such a section defines an arithmetically Gorenstein zero-scheme of degree 5, which clearly contains $Z$ and so links $Z$ to one point.

Remark 5.1. A natural question is whether the construction introduced in this paper gives all arithmetically Gorenstein subschemes of $\mathbb{P}^{n}$. We now show that this is not the case, with an example of an arithmetically Gorenstein zeroscheme in $\mathbb{P}^{3}$ which cannot be produced in this way. Let $P$ be the point defined by the ideal $I=(x, y, z)$ in $k[w, x, y, z]$. Let $Z$ be the scheme defined by the saturated ideal $I^{2}$. Notice that $\operatorname{deg} Z=4$ and that $I^{2}$ is generated by six quadrics. Since $I$ involves only the variables $x, y$ and $z$, the same is true of $I^{2}$, and hence of the syzygy matrix of $I^{2}$ (regardless of the choice of generators for $I^{2}$ ). It follows from [37], Theorem 2.1 , that no five quadrics can define $Z$ scheme-theoretically.

Let $J=\left(x^{2}, y^{2}, z^{2}, x y-y z, x z-y z\right) \subset I^{2}$. From above, $J$ defines a scheme $Y$ which contains $Z$ but is not equal to $Z$. Linking by the complete intersection $\left(x^{2}, y^{2}, z^{2}\right)$, one can check that the residual $Y^{\prime}$ has ideal $J^{\prime}=\left(x+y+z, x^{2}, y^{2}, z^{2}\right)=$ $\left(x+y+z, y^{2}, y z, z^{2}\right)$. This is a saturated ideal defining a degenerate scheme of degree 3 , also supported on $P$. Its minimal free resolution is

$$
0 \rightarrow 2 S(-4) \rightarrow 5 S(-3) \rightarrow S(-1) \oplus 3 S(-2) \rightarrow J^{\prime} \rightarrow 0 .
$$

One can then apply Ferrand's mapping cone construction [34] to get a free resolution of the original scheme $Y$; and splitting off the three free summands which correspond to the fact that $x^{2}, y^{2}$ and $z^{2}$ are all minimal generators of $J^{\prime}$, we get that $Y$ is arithmetically Gorenstein (i.e. that the rank of the last free module in the resolution is 1 ).

We now claim that $J$ cannot be constructed using our procedure. It is too small to be produced by any matrix of size $2 \times 5$ or larger (with a few exceptions that can be checked individually), so it is enough to show that it cannot be obtained by starting with a $1 \times 4$ matrix of linear forms. The key observation is that any scheme produced by the method of this paper, starting with a $1 \times 4$ matrix, is defined scheme-theoretically by four polynomials. Hence it suffices to show that our zero-scheme $Y$ cannot be defined as a scheme by four polynomials. We use two results from Portelli and Spangher [37]. First, their Theorem 3.1 says that it is enough to show that no four quadrics in $I$ define $Z$ (i.e. we do not have to consider forms of higher degree). Then a second use of their Theorem 2.1 allows us to conclude the desired result.

Remark 5.2. It follows from [37] exactly as above that if $I$ is the ideal of any point in $\mathbb{P}^{n}$, and if $Z$ is the scheme defined by $I^{k}$ (which is a saturated ideal), then $Z$ is defined by $\frac{n-1+k}{n-1}$ forms of degree $k$, and it is not scheme-theoretically defined by any smaller number of homogeneous polynomials. A similar statement can be made for any linear space. As a consequence, if $Z$ is any reduced set of points in $\mathbb{P}^{n}$ with homogeneous ideal $I$, and if $Z^{(k)}$ is the scheme defined by the $k$ th symbolic 
power $I^{(k)}$ (the saturation of $I^{k}$ ), then $Z^{(k)}$ cannot be cut out scheme-theoretically by fewer than $\frac{n-1+k}{n-1}$ forms.

5.2. Application to constructing threefolds in $\mathbb{P}^{6}$. As mentioned earlier, liaison theory in codimension two has been a powerful tool in the construction of smooth curves in $\mathbb{P}^{3}$, smooth surfaces in $\mathbb{P}^{4}$, and smooth threefolds in $\mathbb{P}^{5}$. In [13] Ellingsrud and Peskine proved that the number of families of smooth surfaces not of general type is bounded in $\mathbb{P}^{4}$. In [8] this result was extended to show that the number of families of smooth threefolds not of general type is bounded in $\mathbb{P}^{5}$. This leads to the exciting possibility of a complete classification of all smooth codimension two varieties not of general type in $\mathbb{P}^{4}$ and $\mathbb{P}^{5}$. One can use a variety of techniques to limit various numerical invariants of such projective manifolds, but the actual construction of examples is often very difficult. Liaison theory has proven to be a powerful tool in the construction of examples, hence in establishing the existence of certain families.

A sweeping conjecture [43] that has a certain intuitive appeal is that the number of families of nongeneral type smooth $k$-folds in $\mathbb{P}^{n}$ is bounded provided $n \leq 2 k$. If this conjecture is established, then a complete classification of the families would be exciting indeed. The first case one might consider in codimension three is the case of threefolds in $\mathbb{P}^{6}$. Okonek has used the techniques of classical liaison theory to produce many families of low degree threefolds in $\mathbb{P}^{6}$ (without the restriction of nongeneral type). As an application of Gorenstein linkage, we construct two low degree, locally Cohen-Macaulay threefolds that do not appear on Okonek's list. The first example illustrates the construction technique for Gorenstein schemes presented in this paper. The second example illustrates the advantage of constructing Gorenstein schemes as sections of sheaves by combining the construction with Gorenstein linkage theory.

Example 5.3. Let $R=k\left[x_{0}, \ldots, x_{6}\right]$. Let $A$ be a $2 \times 5$ matrix of linear forms from $R$ such that the ideal of all $2 \times 2$ minors of $A$ defines a scheme of codimension 4 in $\mathbb{P}^{6}$. View the matrix $A$ as giving a map from $F=\bigoplus_{i=1}^{5} R(-1)$ to $G=\bigoplus_{i=1}^{2} R$. Let $\mathcal{Q}$ denote the sheafification of the kernel of the map defined by $A$. By the Buchsbaum-Rim complex, $\mathcal{Q}(3)$ is globally generated. Let $s$ be a section of $\mathcal{Q}(4)$ which vanishes on a codimension three scheme $S$. Let $X$ be the pure codimension three component of $S$. Then $X$ is a smooth arithmetically Gorenstein threefold with degree 14 and sectional genus 15 . If $I_{X}$ denotes the homogeneous ideal of $X$, then $I_{X}$ has the minimal free resolution

$$
0 \rightarrow R(-7) \rightarrow \bigoplus_{i=1}^{7} R(-4) \rightarrow \bigoplus_{i=1}^{7} R(-3) \rightarrow I_{X} \rightarrow 0
$$

(The smoothness of $X$ follows from [32], Proposition 2.1.)

Example 5.4. Let $H$ be a linear threefold defined by three hyperplanes in $\mathbb{P}^{6}$. Let $A$ and $\mathcal{Q}$ be as described in the previous example. There exist sections of $\mathcal{Q}(4)$ containing $H$; hence there exists an arithmetically Gorenstein scheme, $X$, of degree 14 and sectional genus 15 containing $H$. Using $X$, we link $H$ to a threefold $T$ with degree 13 and sectional genus 12. If $I_{T}$ denotes the homogeneous ideal of $T$, then 
$I_{T}$ has the minimal free resolution

$$
0 \rightarrow \bigoplus_{i=1}^{3} R(-6) \rightarrow \bigoplus_{i=1}^{7} R(-4) \oplus \bigoplus_{i=1}^{3} R(-5) \rightarrow \bigoplus_{i=1}^{7} R(-3) \oplus R(-4) \rightarrow I_{T} \rightarrow 0 .
$$

It will be an important problem to determine conditions for which Gorenstein linkage will produce a smooth residual. Many examples of low degree locally CohenMacaulay threefolds in $\mathbb{P}^{6}$ can be quickly produced in the manner illustrated above. It remains to determine which of the examples are smooth.

5.3. Higher codimension. One is naturally interested in extensions of Theorem 4.1 to higher codimensions. In even codimension there does not seem to be a direct analog to Theorem 4.1. For example, let $X$ denote the vanishing locus of a section of some twist of the cotangent bundle in $\mathbb{P}^{2 n}$ (this is a natural analog to the situation of Corollary 1.2). $X$ will be a zero-scheme in $\mathbb{P}^{2 n}$ but will not be arithmetically Gorenstein. A surprising fact is that $X$ will be an almost complete intersection, i.e a scheme whose saturated ideal has one more generator than its codimension. Kunz [26] has shown that almost complete intersections are never arithmetically Gorenstein. But all is not lost: by linking with a complete intersection one can obtain an arithmetically Gorenstein zero-scheme as the residual. This certainly suggests several problems worth pursuing, and we summarize it as follows.

Problem 5.5. Let $A$ be a $t \times(t+2 r)$ homogeneous matrix over $R=k\left[x_{0}, \ldots, x_{n}\right]$ such that the ideal of $t \times t$ minors of $A$ defines a scheme of codimension $2 r+1$. View $A$ as defining a map between two free $R$-modules $F$ and $G$. Let $Q$ denote the kernel of this map. Sheafify $Q$ to obtain a rank $2 r$ reflexive sheaf $\mathcal{Q}$. Let $s$ be a section of $\mathcal{Q}$ which has a codimension $2 r$ degeneracy locus, $S$. Let $I_{X}$ denote the homogeneous ideal of the pure codimension $2 r$ component of $S$. What is the minimal free resolution of $I_{X}$ ? Can we utilize $I_{X}$ to construct arithmetically Gorenstein schemes of codimension $2 r$ ?

It seems that the case of odd codimension is much cleaner than that of even codimension. Indeed, a wonderful generalization of Theorem 4.1 appears to hold. We have worked out most of the details in the case of codimension 5. Full details will appear in a forthcoming paper. For now we state the following as conjectures.

Conjecture 5.6. Let $A$ be a $t \times(t+5)$ homogeneous matrix over $R$ such that the ideal of $t \times t$ minors of $A$ defines a scheme of codimension six. View $A$ as defining a map between two free $R$-modules $F$ and $G$. Let $Q$ denote the kernel of this map. Sheafify $Q$ to obtain a rank five reflexive sheaf $\mathcal{Q}$. Let $s$ be a section of $\mathcal{Q}$ which has a codimension five degeneracy locus, $S$. Let $I_{X}$ denote the homogeneous ideal of the pure codimension five component of $S$. Let $c_{1}$ denote the first Chern class of $\mathcal{Q}$. Then $I_{X}$ is arithmetically Gorenstein with free resolution

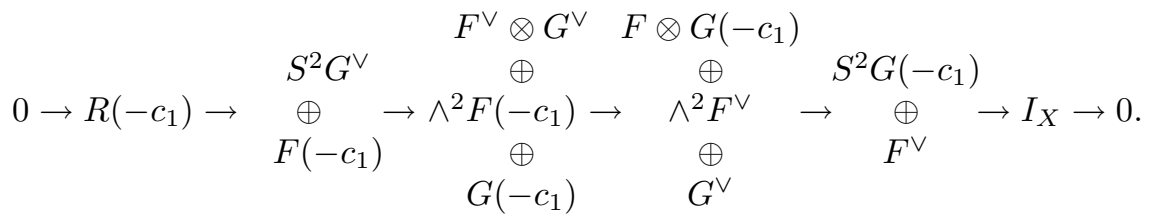


Notice that just as in Theorem 4.1, there is a striking symmetry in the components of this resolution.

A corollary to this conjecture takes on a particularly nice form in $\mathbb{P}^{5}$.

Corollary 5.7. Let $\mathcal{E}$ be a rank five vector bundle in $\mathbb{P}^{5}$ such that $H^{i}\left(\mathbb{P}^{5}, \mathcal{E}(n)\right)=0$ for all $n$ and for $i=2,3$, and 4 . Let $s$ be a section of $\mathcal{E}$ vanishing on a codimension five scheme $X$. Then $X$ is arithmetically Gorenstein.

Since we have not worked out the details of the resolution in codimension seven or higher, we present a less specific version of the conjecture in this case.

Conjecture 5.8. Let $A$ be $a t \times(t+2 r+1)$ homogeneous matrix over $R$ such that the ideal of $t \times t$ minors of $A$ defines a scheme of codimension $2 r+2$. View $A$ as defining a map between two free $R$-modules $F$ and $G$. Let $Q$ denote the kernel of this map. Sheafify $Q$ to obtain a rank $2 r+1$ reflexive sheaf $\mathcal{Q}$. Let $s$ be a section of $\mathcal{Q}$ which has a codimension $2 r+1$ degeneracy locus, $S$. Let $I_{X}$ denote the homogeneous ideal of the pure codimension $2 r+1$ component of $S$. Let $c_{1}$ denote the first Chern class of $\mathcal{Q}$. Then $I_{X}$ is arithmetically Gorenstein and has a predictable free resolution with final term $R\left(-c_{1}\right)$.

The authors plan to address some of these problems and conjectures in a forthcoming paper with U. Nagel.

\section{REFERENCES}

[1] E. Ballico, G. Bolondi and J. Migliore, The Lazarsfeld-Rao Problem for Liaison Classes of Two-Codimensional Subschemes of $\mathbb{P}^{n}$, Amer. J. of Math. 113 (1991), 117-128. MR 92c: 14047

[2] D. Bayer and M. Stillman, Macaulay, a computer system for computing in Commutative Algebra and Algebraic Geometry.

[3] M.C. Beltrametti, M. Schneider and A. Sommese, Threefolds of degree 9 and 10 in $\mathbb{P}^{5}$, Math. Ann. 288 (1990), 413-444. MR 91m:14060

[4] - The threefolds of degree 11 in $\mathbb{P}^{5}$, Complex Projective Geometry, Lond. Math. Soc. Lecture Note Series, 179 (1992), 59-80. MR 94d:14037

[5] G. Bolondi, Surfaces in $\mathbb{P}^{4}$ and deficiency modules, in the proceedings of the conference "Classification of Algebraic Varieties" (L'Aquila 1992), Contemporary Mathematics 162 (1994) (AMS publ.), 49-63. MR 95c:14036

[6] G. Bolondi and J. Migliore, Configurations of Linear Projective Subvarieties, in "Algebraic Curves and Projective Geometry, Proceedings (Trento, 1988)," Lecture Notes in Mathematics, vol. 1389, Springer-Verlag (1989), 19-31. MR 90i:14053

[7] _ The Lazarsfeld-Rao property on an arithmetically Gorenstein variety, manuscripta math. 78 (1993), 347-368. MR 94a:13008

[8] R. Braun, G. Ottaviani, M. Schneider and F.-O. Schreyer, Boundedness of nongeneral type 3-folds in $\mathbb{P}^{5}$, in "Complex Analysis and Geometry," ed. by V. Ancona and A. Silva (1993), Plenum Press, New York, 311-338. MR 94b:14033

[9] D. Buchsbaum and D. Eisenbud, Algebra structures for finite free resolutions and some structure theorems for ideals of codimension three, Amer. J. Math. 99 (1977), 447-485. MR 56:11983

[10] E. Davis, A.V. Geramita and F. Orecchia, Gorenstein Algebras and the Cayley-Bacharach Theorem, Proc. AMS 93 (1985), 593-597. MR 86k:14034

[11] W. Decker, L. Ein and F. Schreyer, Construction of Surfaces in $\mathbb{P}^{4}$, J. Alg. Geom. 2 (1993), 185-237. MR 94a: 14037

[12] W. Decker and S. Popescu, On Surfaces in $\mathbb{P}^{4}$ and 3-folds in $\mathbb{P}^{5}$, in Vector Bundles in Algebraic Geometry, London Math. Soc. Lecture Note Ser., vol. 208, Cambridge Univ. Press, 1995, pp. 69-100. MR 96d:14046

[13] G. Ellingsrud and C. Peskine, Sur les surfaces lisses de $\mathbb{P}^{4}$, Invent. Math. 95 (1989), 1-11. MR 89j: 14023 
[14] E.G. Evans and P. Griffith, The Syzygy Problem, Ann. Math. 114 (1981) 323-333. MR 83i: 13006

[15] A.V. Geramita and J. Migliore, Hyperplane Sections of a Smooth Curve in $\mathbb{P}^{3}$, Comm. Alg. 17 (1989), 3129-3164. MR 90k:14027

[16] A.V. Geramita and J. Migliore, Reduced Gorenstein Codimension Three Subschemes of Projective Space, Proc. Amer. Math. Soc. 125 (1997), 943-950.

[17] P. Griffiths and J. Harris, Residues and zero-cycles on algebraic varieties, Ann. Math. 108 (1978), 461-505. MR 80d:14006

[18] L. Gruson, R. Lazarsfeld and C. Peskine On a theorem of Castelnuovo, and the equations defining space curves, Invent. Math. 72 (1983) 491-506. MR 85g:14033

[19] R. Hartshorne, "Algebraic Geometry," Springer-Verlag, Graduate Texts in Mathematics 52 (1977). MR 57:3116

[20] _ Stable Reflexive Sheaves, Math. Ann. 254 (1980) 121-176. MR 82b:14011

[21] appear).

[22] C. Huneke, Numerical invariants of liaison classes, Invent. math. 75 (1984), 301-325. MR 85i: 13021

[23] C. Huneke, B. Ulrich, General Hyperplane Sections of Algebraic Varieties, J. Alg. Geom. 2 (1993), 487-505. MR 94b:14046

[24] M. Kreuzer, On 0-dimensional complete intersections, Mathematische Annalen 292 (1992), 43-58. MR 92m:14064

[25] M. Kreuzer, "Vektorbündel und der Satz von Cayley-Bacharach," Dissertation, Regensburger Math. Schr., vol. 21, Universität Regensburg 1989. MR 91d:14035

[26] E. Kunz, Almost Complete Intersections are not Gorenstein Rings, J. Alg. 28 (1974), 111115. MR 48:8496

[27] R. Maggioni and A. Ragusa, The Hilbert Function of Generic Plane Sections of Curves in $\mathbb{P}^{3}$, Inv. Math. 91 (1988), 253-258. MR 89g:14027

[28] M. Martin-Deschamps and D. Perrin, Sur la Classification des Courbes Gauches, Astérisque 184-185, Soc. Math. de France (1990). MR 91h:14039

[29] R. Miró-Roig, On the Degree of Smooth non-Arithmetically Cohen-Macaulay Threefolds $\mathbb{P}^{5}$, Proc. A.M.S. 110 (1990), 311-313. MR 91c: 14051

[30] J. Migliore, Curves and their Hyperplane Sections, lecture notes for the Notre Dame Algebraic Geometry Seminar (1989).

[31] Juan Migliore, "An Introduction to Deficiency Modules and Liaison Theory for Subschemes of Projective Space," (monograph) Global Analysis Research Center, Seoul National University, Lecture Notes Series No. 24 (1994). MR 95k:14070

[32] C. Okonek, Notes on Varieties of Codimension 3 in $\mathbb{P}^{N}$, manuscripta math. 84 (1994), 421-442. MR 95k:14071

[33] C. Okonek, M. Schneider and H. Spindler, "Vector Bundles on Complex Projective Space," Birkhauser, Progress in Mathematics 3 (1980). MR 81b:14001

[34] C. Peskine and L. Szpiro, Liaison des variétés algébriques. I, Inv. Math. 26 (1974), 271-302. MR 51:526

[35] C. Peterson, Applications of liaison theory to schemes supported on lines, growth of the deficiency module, and low rank vector bundles, Ph.D. thesis, Duke University (1994).

[36] S. Popescu and K. Ranestad, Surfaces of degree 10 in Projective Fourspace via Linear Systems and Linkage, J. Alg. Geom. 5 (1996), 13-76. MR 96i:14002

[37] D. Portelli and W. Spangher, On the equations which are needed to define a closed subscheme of the projective space, J. Pure and Applied Algebra 98 (1995), 83-93. MR 96d:14002

[38] K. Ranestad, Surfaces of degree 10 in the projective fourspace, in "Problems in the Theory of Surfaces and their Classification, Cortona, Italy, 1988," ed. by F. Catanese and C. Ciliberto, Sympos. Math. 32 (1991), 270-307, INDAM, Academic Press, London. MR 95c:14005

[39] P. Rao, Liaison among Curves in $\mathbb{P}^{3}$, Invent. Math. 50 (1979), 205-217. MR 80e:14023

[40] _ Liaison Equivalence Classes, Math. Ann. 258 (1981), 169-173. MR 83j:14045

[41] R. Re, Sulle sezioni iperpiane di una varietá proiettiva, Le Matematiche (Catania) 42 (1987), 211-218 (1989). MR 90j:14069

[42] P. Schenzel, Notes on Liaison and Duality, J. Math. of Kyoto Univ. 22 (1982), 485-498. MR 84a: 13012 
[43] M. Schneider, Boundedness of low-codimensional submanifolds of projective space, Int. J. Math. 3 (3) (1992), 397-399. MR 93g:14057

[44] C. Weibel, "An introduction to homological algebra," Cambridge University Press, Cambridge studies in advanced mathematics 38 (1994). MR 95f: 18001

Department of Mathematics, University of Notre Dame, Notre Dame, Indiana 46556

E-mail address: Juan.C.Migliore.1@nd.edu

Department of Mathematics, University of Notre Dame, Notre Dame, Indiana 46556

E-mail address: peterson@math.nd.edu 